\title{
TWO-TERM SPECTRAL ASYMPTOTICS FOR THE DIRICHLET LAPLACIAN ON A BOUNDED DOMAIN
}

\author{
RUPERT L. FRANK \\ Department of Mathematics, Princeton University \\ Washington Road, Princeton, NJ 08544, USA \\ E-mail: rlfrank@math.princeton.edu \\ LEANDER GEISINGER \\ Fachbereich Mathematik und Physik, Universität Stuttgart \\ Pfaffenwaldring 57, 70569 Stuttgart, Germany \\ E-mail: geisinger@mathematik.uni-stuttgart.de \\ Dedicated to Ari Laptev on the occasion of his 60th birthday

\begin{abstract}
Let $-\Delta$ denote the Dirichlet Laplace operator on a bounded open set in $\mathbb{R}^{d}$. We study the sum of the negative eigenvalues of the operator $-h^{2} \Delta-1$ in the semiclassical limit $h \rightarrow 0+$. We give a new proof that yields not only the first term of the asymptotic formula but also the second term involving the surface area of the boundary of the set. The proof is valid under weak smoothness assumptions on the boundary.
\end{abstract} \\ Keywords: Dirichlet Laplace operator; semiclassical limit; Weyl's law.
}

\section{Introduction and main result}

\subsection{Introduction}

Let $\Omega$ be a bounded open set in $\mathbb{R}^{d}, d \geq 2$. We consider the Dirichlet Laplace operator $-\Delta_{\Omega}$ defined as a self-adjoint operator in $L^{2}(\Omega)$ generated by the form

$$
\left(v,-\Delta_{\Omega} v\right)=\int_{\Omega}|\nabla v(x)|^{2} d x
$$

with form domain $H_{0}^{1}(\Omega)$. Since $\Omega$ is bounded the embedding of $H_{0}^{1}(\Omega)$ into $L^{2}(\Omega)$ is compact and the spectrum of $-\Delta_{\Omega}$ is discrete. It consists of a series of positive eigenvalues $0<\lambda_{1} \leq \lambda_{2} \leq \cdots$ accumulating at infinity only.

In general, the eigenvalues $\lambda_{k}$ cannot be calculated explicitly and especially for large $k$ it is difficult to evaluate them numerically. Therefore it is interesting to describe the asymptotic behavior of $\lambda_{k}$ as $k \rightarrow \infty$. This is equivalent to the asymptotics of the negative eigenvalues of the operator

(C) 2010 by the authors. This paper may be reproduced, in its entirety, for non-commercial purposes. 


$$
H_{\Omega}=-h^{2} \Delta_{\Omega}-1
$$

in the semiclassical limit $h \rightarrow 0+$.

The first general result is due to $\mathrm{H}$. Weyl who studied the counting function

$$
N_{\Omega}(h)=\sharp\left\{\lambda_{k}<h^{-2}\right\}=\operatorname{Tr}\left(H_{\Omega}\right)_{-}^{0} .
$$

In 1912 he showed that the first term of its semiclassical limit is given by the phase-space volume [11]: For any open bounded set $\Omega \subset \mathbb{R}^{d}$ the limit

$$
N_{\Omega}(h)=C_{d}|\Omega| h^{-d}+o\left(h^{-d}\right)
$$

holds as $h \rightarrow 0+$, where

$$
C_{d}=\frac{1}{(2 \pi)^{d}} \int_{\mathbb{R}^{d}}\left(|p|^{2}-1\right)_{-}^{0} d p=\frac{\omega_{d}}{(2 \pi)^{d}}
$$

and $\omega_{d}$ denotes the volume of the unit ball in $\mathbb{R}^{d}$.

H. Weyl conjectured in [12] that this formula can be refined by a second term of order $h^{-d+1}$ depending on the boundary of $\Omega$. This stimulated a detailed analysis of the semiclassical limit of partial differential operators. We refer to the books $[4,6,9]$ for general results and an overview over the literature. Eventually, the existence of a second term was proved by V. Ivrii by means of a detailed microlocal analysis [5]: If the boundary of $\Omega$ is smooth and if the measure of all periodic geodesic billiards is zero then the limit

$$
N_{\Omega}(h)=C_{d}|\Omega| h^{-d}-\frac{1}{4} C_{d-1}|\partial \Omega| h^{-d+1}+o\left(h^{-d+1}\right)
$$

holds as $h \rightarrow 0+$, where $|\partial \Omega|$ denotes the surface area of the boundary.

In this article we are interested in the sum of the negative eigenvalues

$$
\operatorname{Tr}\left(H_{\Omega}\right)_{-}=\sum\left(h^{2} \lambda_{k}-1\right)_{-} .
$$

This quantity describes the energy of non-interacting, fermionic particles trapped in $\Omega$ and plays an important role in physical applications.

The asymptotic relation (1) immediately implies a refined formula for the semiclassical limit of $\operatorname{Tr}\left(H_{\Omega}\right)_{-}$: Suppose that the aforementioned geometric conditions on $\Omega$ are satisfied. Then integrating (1) yields

$$
\operatorname{Tr}\left(H_{\Omega}\right)_{-}=L_{d}|\Omega| h^{-d}-\frac{1}{4} L_{d-1}|\partial \Omega| h^{-d+1}+o\left(h^{-d+1}\right)
$$

as $h \rightarrow 0+$, with

$$
L_{d}=\int_{\mathbb{R}^{d}}\left(|p|^{2}-1\right)_{-} d p=\frac{2}{d(d+2)} \frac{\omega_{d}}{(2 \pi)^{d}} .
$$

In the following we present a direct approach to derive the semiclassical limit of $\operatorname{Tr}\left(H_{\Omega}\right)_{-}$. We prove (2) without using the result for the counting function. Since we do not apply any microlocal methods the proof works under much weaker conditions. 


\subsection{Main result}

Our main result holds without any global geometric conditions on $\Omega$. We only require weak smoothness conditions on the boundary - namely that the boundary belongs to the class $C^{1, \alpha}$ for some $\alpha>0$. That means, we assume that the local charts of $\Omega$ are differentiable and the derivatives are Hölder continuous with exponent $\alpha$.

Theorem 1.1. Let the boundary of $\Omega$ satisfy $\partial \Omega \in C^{1, \alpha}, 0<\alpha \leq 1$. Then the asymptotic limit

$$
\operatorname{Tr}\left(H_{\Omega}\right)_{-}=L_{d}|\Omega| h^{-d}-\frac{1}{4} L_{d-1}|\partial \Omega| h^{-d+1}+O\left(h^{-d+1+\alpha /(2+\alpha)}\right)
$$

holds as $h \rightarrow 0+$.

Our work was stimulated by the question whether similar two-term formulae hold for non-local, non-smooth operators. This is unknown, since the microlocal methods leading to (1) are not applicable. Therefore it is necessary to use a direct approach.

Indeed, Theorem 1.1 can be extended to fractional powers of the Dirichlet Laplace operator [3]. The strategy of the proof is similar but dealing with non-local operators is more difficult and elaborate. In order to give a flavor of our techniques we confine ourselves in this article to the local case.

The question whether the second term of the semiclassical limit of $\operatorname{Tr}\left(H_{\Omega}\right)_{-}$ exists for Lipschitz domains $\Omega$ remains open.

\subsection{Strategy of the proof}

The proof of Theorem 1.1 is divided into three steps: First, we localize the operator $H_{\Omega}$ into balls, whose size varies depending on the distance to the complement of $\Omega$. Then we analyze separately the semiclassical limit in the bulk and at the boundary.

To localize, let $d(u)=\inf \{|x-u|: x \notin \Omega\}$ denote the distance of $u \in \mathbb{R}^{d}$ to the complement of $\Omega$. We set

$$
l(u)=\frac{1}{2}\left(1+\left(d(u)^{2}+l_{0}^{2}\right)^{-1 / 2}\right)^{-1},
$$

where $0<l_{0} \leq 1$ is a parameter depending only on $h$. Indeed, we will finally choose $l_{0}$ proportional to $h^{2 /(\alpha+2)}$.

In Section 3 we introduce real-valued functions $\phi_{u} \in C_{0}^{\infty}\left(\mathbb{R}^{d}\right)$ with support in the ball $B_{u}=\left\{x \in \mathbb{R}^{d}:|x-u|<l(u)\right\}$. For all $u \in \mathbb{R}^{d}$ these functions satisfy

$$
\left\|\phi_{u}\right\|_{\infty} \leq C, \quad\left\|\nabla \phi_{u}\right\|_{\infty} \leq C l(u)^{-1}
$$

and for all $x \in \mathbb{R}^{d}$

$$
\int_{\mathbb{R}^{d}} \phi_{u}^{2}(x) l(u)^{-d} d u=1 .
$$

Here and in the following the letter $C$ denotes various positive constants that might depend on $\Omega$, but that are independent of $u, l_{0}$ and $h$.

Proposition 1.1. For $0<l_{0} \leq 1$ and $h>0$ we have

$$
\left|\operatorname{Tr}\left(H_{\Omega}\right)_{-}-\int_{\mathbb{R}^{d}} \operatorname{Tr}\left(\phi_{u} H_{\Omega} \phi_{u}\right)_{-} l(u)^{-d} d u\right| \leq C l_{0}^{-1} h^{-d+2} .
$$


In view of this result, one can analyze the local asymptotics, i. e., the asymptotic

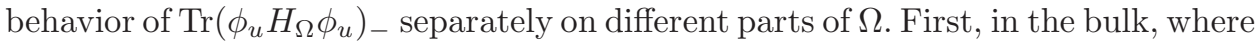
the influence of the boundary is not felt.

Proposition 1.2. Assume that $\phi \in C_{0}^{\infty}(\Omega)$ is supported in a ball of radius $l>0$ and that

$$
\|\nabla \phi\|_{\infty} \leq C l^{-1}
$$

is satisfied. Then for $h>0$ the estimate

$$
\left|\operatorname{Tr}\left(\phi H_{\Omega} \phi\right)_{-}-L_{d} \int_{\Omega} \phi^{2}(x) d x h^{-d}\right| \leq C l^{d-2} h^{-d+2}
$$

holds, with a constant depending only on the constant in (5).

Close to the boundary of $\Omega$, more precisely, if the support of $\phi$ intersects the boundary, a term of order $h^{-d+1}$ appears:

Proposition 1.3. Assume that $\phi \in C_{0}^{\infty}\left(\mathbb{R}^{d}\right)$ is supported in a ball of radius $l>0$ intersecting the boundary of $\Omega$ and that inequality (5) is satisfied.

Then for all $0<l \leq 1$ and $0<h \leq 1$ the estimate

$$
\left|\operatorname{Tr}\left(\phi H_{\Omega} \phi\right)_{-}-L_{d} \int_{\Omega} \phi^{2}(x) d x h^{-d}+\frac{1}{4} L_{d-1} \int_{\partial \Omega} \phi^{2}(x) d \sigma(x) h^{-d+1}\right| \leq r(l, h)
$$

holds. Here $d \sigma$ denotes the $d-1$-dimensional volume element of $\partial \Omega$ and the remainder satisfies

$$
r(l, h) \leq C\left(\frac{l^{d-2}}{h^{d-2}}+\frac{l^{2 \alpha+d-1}}{h^{d-1}}+\frac{l^{d+\alpha}}{h^{d}}\right)
$$

with a constant depending on $\Omega,\|\phi\|_{\infty}$ and the constant in (5).

Based on these propositions we can complete the proof of the main result.

Proof of Theorem 1.1. In order to apply Proposition 1.3 to the operators $\phi_{u} H_{\Omega} \phi_{u}$, we need to estimate $l(u)$ uniformly. Assume that $u \in \mathbb{R}^{d}$ satisfies $B_{u} \cap \partial \Omega \neq \emptyset$. Then we have $d(u) \leq l(u)$, which by definition of $l(u)$ implies

$$
l(u) \leq l_{0} / \sqrt{3} .
$$

In view of (3) we can therefore apply Proposition 1.2 and Proposition 1.3 to all functions $\phi_{u}, u \in \mathbb{R}^{d}$. Combining these results with Proposition 1.1 we get

$$
\begin{aligned}
& \left|\operatorname{Tr}\left(H_{\Omega}\right)_{-}-\frac{L_{d}}{h^{d}} \int_{\mathbb{R}^{d}} \int_{\Omega} \phi_{u}^{2}(x) d x \frac{d u}{l(u)^{d}}+\frac{L_{d-1}}{4 h^{d-1}} \int_{\mathbb{R}^{d}} \int_{\partial \Omega} \phi_{u}^{2}(x) d \sigma(x) \frac{d u}{l(u)^{d}}\right| \\
& \leq C\left(l_{0}^{-1} h^{-d+2}+\int_{U_{1}} l(u)^{-2} d u h^{-d+2}+\int_{U_{2}} r(l(u), h) l(u)^{-d} d u\right),
\end{aligned}
$$


where $U_{1}=\left\{u \in \Omega: B_{u} \cap \partial \Omega=\emptyset\right\}$ and $U_{2}=\left\{u \in \mathbb{R}^{d}: B_{u} \cap \partial \Omega \neq \emptyset\right\}$. Now we change the order of integration and by virtue of (4) we obtain

$$
\begin{aligned}
& \left|\operatorname{Tr}\left(H_{\Omega}\right)_{-}-L_{d}\right| \Omega\left|h^{-d}+\frac{1}{4} L_{d-1}\right| \partial \Omega\left|h^{-d+1}\right| \\
& \leq C\left(l_{0}^{-1} h^{-d+2}+\int_{U_{1}} l(u)^{-2} d u h^{-d+2}+\int_{U_{2}} r(l(u), h) l(u)^{-d} d u\right) .
\end{aligned}
$$

It remains to estimate the remainder terms. Note that, by definition of $l(u)$, we have

$$
l(u) \geq \frac{1}{4} \min (d(u), 1) \quad \text { and } \quad l(u) \geq \frac{l_{0}}{4}
$$

for all $u \in \mathbb{R}^{d}$. Together with (6) this implies

$$
\int_{U_{1}} l(u)^{-2} d u \leq C l_{0}^{-1} \text { and } \int_{U_{2}} l(u)^{a} d u \leq C l_{0}^{a} \int_{\left\{d(u) \leq l_{0}\right\}} d u \leq C l_{0}^{a+1}
$$

for any $a \in \mathbb{R}$. Inserting these estimates into (7) we find that the remainder terms are bounded from above by a constant times

$$
l_{0}^{-1} h^{-d+2}+l_{0}^{2 \alpha} h^{-d+1}+l_{0}^{\alpha+1} h^{-d} .
$$

Finally, we choose $l_{0}$ proportional to $h^{2 /(\alpha+2)}$ and conclude that all error terms in (7) equal $O\left(h^{-d+1+\alpha /(2+\alpha)}\right)$ as $h \rightarrow 0+$.

The remainder of the text is structured as follows. In Section 2 we analyze the local asymptotics and outline the proofs of Propositions 1.2 and 1.3. In Section 3, we perform the localization and, in particular, prove Proposition 1.1.

\section{Local asymptotics}

To prove the propositions we need the following rough estimate, a variant of the Berezin-Lieb-Li-Yau inequality $[2,7,8]$.

Lemma 2.1. For any $\phi \in C_{0}^{\infty}\left(\mathbb{R}^{d}\right)$ and $h>0$

$$
\operatorname{Tr}\left(\phi H_{\Omega} \phi\right)_{-} \leq L_{d} \int_{\mathbb{R}^{d}} \phi^{2}(x) d x h^{-d} .
$$

Proof. Let us introduce the operator

$$
H_{0}=-h^{2} \Delta-1
$$

defined with form domain $H^{1}\left(\mathbb{R}^{d}\right)$. The variational principle for sums of eigenvalues implies $\operatorname{Tr}\left(\phi H_{\Omega} \phi\right)_{-} \leq \operatorname{Tr}\left(\phi\left(H_{0}\right)_{-} \phi\right)_{-}$. Using the Fourier-transform one can derive an explicit expression for the kernel of $\left(H_{0}\right)_{-}$and inserting this yields the claim. 


\subsection{Local asymptotics in the bulk}

First we assume $\phi \in C_{0}^{\infty}(\Omega)$. Then we have $\operatorname{Tr}\left(\phi H_{\Omega} \phi\right)_{-}=\operatorname{Tr}\left(\phi H_{0} \phi\right)_{-}$, since the form domains of $\phi H_{\Omega} \phi$ and $\phi H_{0} \phi$ coincide. Moreover, by scaling, we can assume $l=1$. Thus, to prove Proposition 1.2, it suffices to establish the estimate

$$
\left|\operatorname{Tr}\left(\phi H_{0} \phi\right)_{-}-L_{d} \int_{\mathbb{R}^{d}} \phi^{2}(x) d x h^{-d}\right| \leq C h^{-d+2}
$$

for $h>0$. The lower bound follows immediately from Lemma 2.1. The upper bound can be derived in the same way as in the proof of Lemma 2.3 below. Indeed, by choosing the trial density matrix $\gamma=\chi\left(H_{0}\right)_{-}^{0} \chi$ we find

$$
\operatorname{Tr}\left(\phi H_{0} \phi\right)_{-} \geq L_{d} \int_{\mathbb{R}^{d}} \phi^{2}(x) d x-C_{d} \int_{\mathbb{R}^{d}}(\nabla \phi)^{2}(x) d x h^{-d+2}
$$

and the claim follows.

\subsection{Straightening the boundary}

Here we transform the operator $H_{\Omega}$ locally to an operator given on the half-space $\mathbb{R}_{+}^{d}=\left\{y \in \mathbb{R}^{d}: y_{d}>0\right\}$. There we define the operator $H^{+}$in the same way as $H_{\Omega}$, with form domain $H_{0}^{1}\left(\mathbb{R}_{+}^{d}\right)$.

Under the conditions of Proposition 1.3 let $B$ denote the open ball of radius $l>0$, containing the support of $\phi$. Choose $x_{0} \in B \cap \partial \Omega$ and let $\nu_{x_{0}}$ be the normed inner normal vector at $x_{0}$. We choose a Cartesian coordinate system such that $x_{0}=0$ and $\nu_{x_{0}}=(0, \ldots, 0,1)$, and we write $x=\left(x^{\prime}, x_{d}\right) \in \mathbb{R}^{d-1} \times \mathbb{R}$ for $x \in \mathbb{R}^{d}$.

For sufficiently small $l>0$ one can introduce new local coordinates near the boundary. Let $D$ denote the projection of $B$ on the hyperplane given by $x_{d}=0$. Since the boundary of $\Omega$ is compact and in $C^{1, \alpha}$, there exists a constant $c>0$, such that for $0<l \leq c$ we can find a real function $f \in C^{1, \alpha}$ given on $D$, satisfying

$$
\partial \Omega \cap B=\left\{\left(x^{\prime}, x_{d}\right): x^{\prime} \in D, x_{d}=f\left(x^{\prime}\right)\right\} \cap B .
$$

The choice of coordinates implies $f(0)=0$ and $\nabla f(0)=0$. Since $f \in C^{1, \alpha}$ and the boundary of $\Omega$ is compact we can estimate

$$
\sup _{x^{\prime} \in D}\left|\nabla f\left(x^{\prime}\right)\right| \leq C l^{\alpha}
$$

with a constant $C>0$ depending only on $\Omega$, in particular independent of $f$.

Now we introduce new local coordinates given by a diffeomorphism $\varphi: D \times \mathbb{R} \rightarrow$ $\mathbb{R}^{d}$. We set $y_{j}=\varphi_{j}(x)=x_{j}$ for $j=1, \ldots, d-1$ and $y_{d}=\varphi_{d}(x)=x_{d}-f\left(x^{\prime}\right)$. Note that the determinant of the Jacobian matrix of $\varphi$ equals 1 and that the inverse of $\varphi$ is defined on $\operatorname{ran} \varphi=D \times \mathbb{R}$. There we define $\tilde{\phi}=\phi \circ \varphi^{-1}$ and extend it by zero to $\mathbb{R}^{d}$, such that $\tilde{\phi} \in C_{0}^{1}\left(\mathbb{R}^{d}\right)$ and $\|\nabla \tilde{\phi}\|_{\infty} \leq C l^{-1}$ holds.

Lemma 2.2. For $0<l \leq c$ and any $h>0$ the estimate

$$
\left|\operatorname{Tr}\left(\phi H_{\Omega} \phi\right)_{-}-\operatorname{Tr}\left(\tilde{\phi} H^{+} \tilde{\phi}\right)_{-}\right| \leq C l^{d+\alpha} h^{-d}
$$

holds. Moreover, we have

$$
\int_{\Omega} \phi^{2}(x) d x=\int_{\mathbb{R}_{+}^{d}} \tilde{\phi}^{2}(y) d y
$$


and

$$
\left|\int_{\partial \Omega} \phi^{2}(x) d \sigma(x)-\int_{\mathbb{R}^{d-1}} \tilde{\phi}^{2}\left(y^{\prime}, 0\right) d y^{\prime}\right| \leq C l^{d-1+2 \alpha} .
$$

Proof. The definition of $\tilde{\phi}$ and the fact $\operatorname{det} J \varphi=1$ immediately give (11). Using (9) we estimate

$$
\int_{\partial \Omega} \phi^{2}(x) d \sigma(x)=\int_{\mathbb{R}^{d-1}} \tilde{\phi}^{2}\left(y^{\prime}, 0\right) \sqrt{1+|\nabla f|^{2}} d y^{\prime} \leq \int_{\mathbb{R}^{d-1}} \tilde{\phi}^{2}\left(y^{\prime}, 0\right) d y^{\prime}+C l^{d-1+2 \alpha}
$$

from which (12) follows.

To prove $(10)$ fix $v \in H_{0}^{1}(\Omega)$ with support in $\bar{B}$. For $y \in \operatorname{ran} \varphi$ put $\tilde{v}(y)=$ $v \circ \varphi^{-1}(y)$ and extend $\tilde{v}$ by zero to $\mathbb{R}^{d}$. Note that $\tilde{v}$ belongs to $H_{0}^{1}\left(\mathbb{R}_{+}^{d}\right)$.

An explicit calculation shows

$$
\left|\left(\tilde{v},-\Delta_{\mathbb{R}_{+}^{d}} \tilde{v}\right)-\left(v,-\Delta_{\Omega} v\right)\right| \leq C l^{\alpha}\left(\tilde{v},-\Delta_{\mathbb{R}_{+}^{d}} \tilde{v}\right) .
$$

Hence, we find

$$
\operatorname{Tr}\left(\phi H_{\Omega} \phi\right)_{-} \leq \operatorname{Tr}\left(\tilde{\phi}\left(-\left(1-C l^{\alpha}\right) h^{2} \Delta_{\mathbb{R}_{+}^{d}}-1\right) \tilde{\phi}\right)_{-} .
$$

Set $\varepsilon=2 C l^{\alpha}$ and assume $l$ to be sufficiently small, so that $0<\varepsilon \leq 1 / 2$ holds. Then

$$
\begin{aligned}
\operatorname{Tr}\left(\phi H_{\Omega} \phi\right)_{-} & \leq \operatorname{Tr}\left(\tilde{\phi}\left(-\left(1-C l^{\alpha}\right) h^{2} \Delta_{\mathbb{R}_{+}^{d}}-1\right) \tilde{\phi}\right)_{-} \\
& \leq \operatorname{Tr}\left(\tilde{\phi}\left(-h^{2} \Delta_{\mathbb{R}_{+}^{d}}-1\right) \tilde{\phi}\right)_{-}+\operatorname{Tr}\left(\tilde{\phi}\left(-\left(\varepsilon-C l^{\alpha}\right) h^{2} \Delta_{\mathbb{R}_{+}^{d}}-\varepsilon\right) \tilde{\phi}\right)_{-} \\
& \leq \operatorname{Tr}\left(\tilde{\phi} H^{+} \tilde{\phi}\right)_{-}+\varepsilon \operatorname{Tr}\left(\tilde{\phi}\left(-\left(h^{2} / 2\right) \Delta_{\mathbb{R}_{+}^{d}}-1\right) \tilde{\phi}\right)_{-} .
\end{aligned}
$$

By Lemma 2.1 we have $\operatorname{Tr}\left(\tilde{\phi}\left(-\left(h^{2} / 2\right) \Delta_{\mathbb{R}_{+}^{d}}-1\right) \tilde{\phi}\right)_{-} \leq C l^{d} h^{-d}$ and we obtain

$$
\operatorname{Tr}\left(\phi H_{\Omega} \phi\right)_{-} \leq \operatorname{Tr}\left(\tilde{\phi} H^{+} \tilde{\phi}\right)_{-}+C l^{d+\alpha} h^{-d} .
$$

Finally, by interchanging the roles of $H_{\Omega}$ and $H^{+}$, we get an analogous upper bound and the proof of Lemma 2.2 is complete.

\subsection{Local asymptotics in half-space}

In view of Lemma 2.2 we can reduce Proposition 1.3 to a statement concerning the operator $H^{+}$, given on the half-space $\mathbb{R}_{+}^{d}$. Indeed, to prove Proposition 1.3, it suffices to establish the following result.

Lemma 2.3. Assume that $\phi \in C_{0}^{1}\left(\mathbb{R}^{d}\right)$ is supported in a ball of radius $l>0$ and that (5) is satisfied. Then for $h>0$ the estimate

$$
\left|\operatorname{Tr}\left(\phi H^{+} \phi\right)_{-}-\frac{L_{d}}{h^{d}} \int_{\mathbb{R}_{+}^{d}} \phi^{2}(x) d x+\frac{L_{d-1}}{4 h^{d-1}} \int_{\mathbb{R}^{d-1}} \phi^{2}\left(x^{\prime}, 0\right) d x^{\prime}\right| \leq C l^{d-2} h^{-d+2}
$$

holds with a constant depending only on the constant in (5). 
Proof. On $\mathbb{R}_{+}^{d}$ we can rescale $\phi$ and assume $l=1$. In a first step we prove the estimate

$$
\begin{aligned}
& \left|\operatorname{Tr}\left(\phi H^{+} \phi\right)_{-}-\frac{L_{d}}{h^{d}} \int_{\mathbb{R}_{+}^{d}} \phi^{2}(x) d x+\int_{\mathbb{R}_{+}^{d}} \phi^{2}(x) \int_{\mathbb{R}^{d}} \cos \left(2 \xi_{d} x_{d} h^{-1}\right)\left(|\xi|^{2}-1\right)_{-} \frac{d \xi d x}{(2 \pi h)^{d}}\right| \\
& \leq C h^{-d+2} .
\end{aligned}
$$

To derive a lower bound we use the inequality $\operatorname{Tr}\left(\phi H^{+} \phi\right)_{-} \leq \operatorname{Tr}\left(\phi\left(H^{+}\right)_{-} \phi\right)$ and diagonalize the operator $\left(H^{+}\right)_{-}$, applying the Fourier-transform in the $x^{\prime}$ coordinates and the sine-transform in the $x_{d}$-coordinate. This yields

$$
\operatorname{Tr}\left(\phi H^{+} \phi\right)_{-} \leq \int_{\mathbb{R}_{+}^{d}} \phi^{2}(x) \int_{\mathbb{R}^{d}} 2 \sin ^{2}\left(\xi_{d} x_{d} h^{-1}\right)\left(|\xi|^{2}-1\right)_{-} \frac{d \xi d x}{(2 \pi h)^{d}}
$$

and the lower bound follows from the identity

$$
2 \sin ^{2}\left(\xi_{d} x_{d} h^{-1}\right)=1-\cos \left(2 \xi_{d} x_{d} h^{-1}\right) .
$$

To prove the upper bound, define the operator $\gamma=\chi\left(H^{+}\right)_{-}^{0} \chi$ with kernel

$$
\gamma(x, y)=\frac{2}{(2 \pi h)^{d}} \chi(x) \int_{|\xi|<1} e^{i \xi^{\prime}\left(x^{\prime}-y^{\prime}\right) / h} \sin \left(\xi_{d} x_{d} h^{-1}\right) \sin \left(\xi_{d} y_{d} h^{-1}\right) d \xi \chi(y),
$$

where $\chi$ denotes the characteristic function of an open ball containing the support of $\phi$. Thus, $\gamma$ is a trace-class operator, satisfying $0 \leq \gamma \leq 1$ and by the variational principle it follows that

$$
\begin{aligned}
& \operatorname{Tr}\left(\phi H^{+} \phi\right)_{-} \\
& \geq-\operatorname{Tr}\left(\gamma \phi H^{+} \phi\right) \\
& =-2 \int_{|\xi|<1}\left(h^{2}\left\|\nabla e^{i \xi^{\prime} \cdot / h} \sin \left(\xi_{d} \cdot h^{-1}\right) \phi\right\|_{L^{2}\left(\mathbb{R}_{+}^{d}\right)}^{2}-\left\|\sin \left(\xi_{d} \cdot h^{-1}\right) \phi\right\|_{L^{2}\left(\mathbb{R}_{+}^{d}\right)}^{2}\right) \frac{d \xi}{(2 \pi h)^{d}} \\
& \geq \int_{\mathbb{R}^{d}}\left(|\xi|^{2}-1\right)_{-} \int_{\mathbb{R}_{+}^{d}} \phi^{2}(x) 2 \sin ^{2}\left(\xi_{d} x_{d} h^{-1}\right) \frac{d x d \xi}{(2 \pi h)^{d}}-C h^{-d+2} .
\end{aligned}
$$

In view of (14) this gives an upper bound and we established (13).

We proceed to analyzing the term in (13) which contains the cosine. We substitute $x_{d}=t h$ and write

$$
\begin{aligned}
& \int_{\mathbb{R}_{+}^{d}} \phi^{2}(x) \int_{\mathbb{R}^{d}} \cos \left(2 \xi_{d} x_{d} h^{-1}\right)\left(|\xi|^{2}-1\right)_{-} \frac{d \xi d x}{(2 \pi h)^{d}} \\
& =\frac{1}{(2 \pi)^{d}} \int_{0}^{\infty} \int_{\mathbb{R}^{d-1}} \phi^{2}\left(x^{\prime}, t h\right) d x^{\prime} \int_{\mathbb{R}^{d}} \cos \left(2 \xi_{d} t\right)\left(|\xi|^{2}-1\right)_{-} d \xi d t h^{-d+1} .
\end{aligned}
$$

Note that

$$
\frac{1}{(2 \pi)^{d}} \int_{0}^{\infty} \int_{\mathbb{R}^{d}} \cos \left(2 \xi_{d} t\right)\left(|\xi|^{2}-1\right)_{-} d \xi d t=\frac{1}{4} L_{d-1} .
$$

Moreover, in $[1,(9.1 .20)]$ it is shown that

$$
\int_{\mathbb{R}^{d}} \cos \left(2 \xi_{d} t\right)\left(|\xi|^{2}-1\right)_{-} d \xi=C \int_{0}^{1} \cos \left(2 \xi_{d} t\right)\left(1-\xi_{d}^{2}\right)^{(d+1) / 2} d \xi_{d}=C \frac{J_{d / 2+1}(2 t)}{t^{d / 2+1}}
$$


where $J_{d / 2+1}$ denotes the Bessel function of the first kind. We remark that $\left|J_{d / 2+1}(2 t)\right|$ is proportional to $t^{d / 2+1}$ as $t \rightarrow 0+$ and bounded by a constant times $t^{-1 / 2}$ as $t \rightarrow \infty$, see [1, (9.1.7) and (9.2.1)]. It follows that

$$
\int_{0}^{\infty} t\left|\int_{\mathbb{R}^{d}} \cos \left(2 \xi_{d} t\right)\left(|\xi|^{2}-1\right)_{-} d \xi\right| d t \leq C \int_{0}^{\infty} t^{-d / 2}\left|J_{d / 2+1}(2 t)\right| d t \leq C .
$$

In view of (15), (16) and (17) we find

$$
\begin{aligned}
& \left|\int_{\mathbb{R}_{+}^{d}} \phi^{2}(x) \int_{\mathbb{R}^{d}} \cos \left(2 \xi_{d} x_{d} h^{-1}\right)\left(|\xi|^{2}-1\right)-\frac{d \xi d x}{(2 \pi h)^{d}}-\frac{L_{d-1}}{4 h^{d-1}} \int_{\mathbb{R}^{d-1}} \phi^{2}\left(x^{\prime}, 0\right) d x^{\prime}\right| \\
& \leq C h^{-d+2} .
\end{aligned}
$$

Inserting this into (13) proves Lemma 2.3.

Proposition 1.3 is a consequence of Lemma 2.2 and Lemma 2.3.

\section{Localization}

Here we construct the family of localization functions $\left(\phi_{u}\right)_{u \in \mathbb{R}^{d}}$ and prove Proposition 1.1. The key idea is to choose the localization depending on the distance to the complement of $\Omega$, see [4, Theorem 17.1.3] and [10].

Fix a real-valued function $\phi \in C_{0}^{\infty}\left(\mathbb{R}^{d}\right)$ with support in $\{|x|<1\}$ and $\|\phi\|_{2}=1$. For $u, x \in \mathbb{R}^{d}$ let $J(x, u)$ be the Jacobian of the map $u \mapsto(x-u) / l(u)$. We define

$$
\phi_{u}(x)=\phi\left(\frac{x-u}{l(u)}\right) \sqrt{J(x, u)} l(u)^{d / 2},
$$

such that $\phi_{u}$ is supported in $\{x:|x-u|<l(u)\}$. According to [10], the functions $\phi_{u}$ satisfy (3) and (4) for all $u \in \mathbb{R}^{d}$.

To prove the upper bound in Proposition 1.1, put

$$
\gamma=\int_{\mathbb{R}^{d}} \phi_{u}\left(\phi_{u} H_{\Omega} \phi_{u}\right)_{-}^{0} \phi_{u} l(u)^{-d} d u
$$

Obviously, $\gamma \geq 0$ holds and in view of (4) also $\gamma \leq 1$. The range of $\gamma$ belongs to $H_{0}^{1}(\Omega)$ and by the variational principle it follows that

$$
-\operatorname{Tr}\left(H_{\Omega}\right)_{-} \leq \operatorname{Tr} \gamma H_{\Omega}=-\int_{\mathbb{R}^{d}} \operatorname{Tr}\left(\phi_{u} H_{\Omega} \phi_{u}\right)_{-} l(u)^{-d} d u .
$$

To prove the lower bound we make use of the IMS-formula

$$
\frac{1}{2}\left(f, \phi^{2}(-\Delta) f\right)+\frac{1}{2}\left(f,-\Delta \phi^{2} f\right)=(f, \phi(-\Delta) \phi f)-\left(f, f(\nabla \phi)^{2}\right),
$$

valid for $\phi \in C_{0}^{\infty}\left(\mathbb{R}^{d}\right)$ and $f \in H_{0}^{1}(\Omega)$. Combining this identity with (4) yields

$$
(f,-\Delta f)=\int_{\mathbb{R}^{d}}\left(\left(f, \phi_{u}(-\Delta) \phi_{u} f\right)-\left(f,\left(\nabla \phi_{u}\right)^{2} f\right)\right) l(u)^{-d} d u .
$$

Using (3) and (4) one can show [10]

$$
\int_{\mathbb{R}^{d}}\left(\nabla \phi_{u}\right)^{2}(x) l(u)^{-d} d u \leq C \int_{\mathbb{R}^{d}} \phi_{u}^{2}(x) l(u)^{-d-2} d u .
$$


We insert this into (18) and deduce

$$
\operatorname{Tr}\left(H_{\Omega}\right)_{-} \leq \int_{\Omega^{*}} \operatorname{Tr}\left(\phi_{u}\left(-h^{2} \Delta-1-C h^{2} l(u)^{-2}\right) \phi_{u}\right)_{-} l(u)^{-d} d u,
$$

where $\Omega^{*}=\left\{u \in \mathbb{R}^{d}: \operatorname{supp} \phi_{u} \cap \Omega \neq \emptyset\right\}$. To estimate the localization error we use Lemma 2.1. For any $u \in \mathbb{R}$, let $\rho_{u}$ be another parameter $0<\rho_{u} \leq 1 / 2$ and estimate

$$
\begin{aligned}
& \operatorname{Tr}\left(\phi_{u}\left(-h^{2} \Delta-1-C h^{2} l(u)^{-2}\right) \phi_{u}\right)_{-} \\
& \leq \operatorname{Tr}\left(\phi_{u}\left(-h^{2} \Delta-1\right) \phi_{u}\right)_{-}+C \operatorname{Tr}\left(\phi_{u}\left(-\rho_{u} h^{2} \Delta-\rho_{u}-h^{2} l(u)^{-2}\right) \phi_{u}\right)_{-} \\
& \leq \operatorname{Tr}\left(\phi_{u} H_{\Omega} \phi_{u}\right)_{-}+C l(u)^{d}\left(\rho_{u} h^{2}\right)^{-d / 2}\left(\rho_{u}+h^{2} l(u)^{-2}\right)^{1+d / 2} .
\end{aligned}
$$

With $\rho_{u}$ proportional to $h^{2} l(u)^{-2}$ we find

$$
\operatorname{Tr}\left(H_{\Omega}\right)_{-} \leq \int_{\Omega^{*}} \operatorname{Tr}\left(\phi_{u} H_{\Omega} \phi_{u}\right)_{-} l(u)^{-d} d u+C h^{-d+2} \int_{\Omega^{*}} l(u)^{-2} d u .
$$

In view of (8) the last integral is bounded by a constant times $l_{0}^{-1}$ and the proof of Proposition 1.1 is complete.

\section{References}

[1] M. Abramowitz and I.A. Stegun, Handbook of Mathematical Functions, Dover Publications, 1972.

[2] F. A. Berezin, Covariant and contravariant symbols of operators, Izv. Akad. Nauk SSSR Ser. Mat. 13 (1972), 1134-1167.

[3] R. L. Frank and L. Geisinger, Refined semiclassical asymptotics for fractional powers of the Laplace operator, in preparation.

[4] L. Hörmander, The analysis of linear partial differential operators III \& IV, SpringerVerlag, Berlin, 1985.

[5] V. Y. Ivrii, On the second term of the spectral asymptotics for the Laplace-Beltrami operator on manifolds with boundary, Funtsional. Anal. i Prilozhen. 14 (1980), no. 2, 25-34.

[6] V. Y. Ivrii, Microlocal analysis and precise spectral asymptotics, Springer Monographs in Mathematics, Springer-Verlag, Berlin, 1998.

[7] P. Li and S. T. Yau, On the Schrödinger equation and the eigenvalue problem, Comm. Math. Phys. 88 (1983), no. 3, 309-318.

[8] E. H. Lieb, The classical limit of quantum spin systems, Comm. Math. Phys. 31 (1973), 327-340.

[9] Y. Safarov and D. Vassiliev, The asymptotic distribution of eigenvalues of partial differential operators, Translations of Mathematical Monographs, 155, American Mathematical Society, Providence, RI, 1997.

[10] J. P. Solovej and W. L. Spitzer, A new coherent states approach to semiclassics which gives Scott's correction, Commun. Math. Phys 241 (2003), 383-420.

[11] H. Weyl, Das asymptotische Verteilungsgesetz der Eigenwerte linearer partieller Diffenertialgleichungen (mit einer Anwendung auf die Theorie der Hohlraumstrahlung), Math. Ann. 71 (1912), no. 4, 441-479.

[12] H. Weyl, Über die Randwertaufgabe der Strahlungstheorie und asymptotische Spektralgesetze, J. Reine Angew. Math. 143 (1913), no. 3, 177-202. 